\title{
Antibiotic stewardship in critically ill patients with suspected ventilator-associated pneumonia
}

\author{
Jumpei Yoshimura ${ }^{1}$, Hideki Hashimoto ${ }^{2,3}$, Kazuma Yamakawa ${ }^{1}$ \\ ${ }^{1}$ Division of Trauma and Surgical Critical Care, Osaka General Medical Center, Sumiyoshi, Osaka, Japan; ${ }^{2}$ Department of Emergency and Critical \\ Care Medicine, ${ }^{3}$ Department of Infectious Diseases, Hitachi General Hospital, Hitachi, Ibaraki, Japan \\ Correspondence to: Kazuma Yamakawa, MD, PhD. Division of Trauma and Surgical Critical Care, Osaka General Medical Center, 3-1-56 Bandai- \\ Higashi, Sumiyoshi, Osaka 558-8558, Japan. Email: kyamakawa-osk@umin.ac.jp. \\ Comment on: Hellyer TP, McAuley DF, Walsh TS, et al. Biomarker-guided antibiotic stewardship in suspected ventilator-associated pneumonia \\ (VAPrapid2): a randomised controlled trial and process evaluation. Lancet Respir Med 2020;8:182-91.
}

Submitted Mar 11, 2020. Accepted for publication Apr 27, 2020.

doi: $10.21037 /$ atm-20-2421

View this article at: http://dx.doi.org/10.21037/atm-20-2421

The rapid spread of antimicrobial resistance is a worldwide problem. A multicenter international prevalence survey revealed that $70 \%$ of patients received some kind of antibiotics during their stay in the intensive care unit (ICU) (1). Because antibiotic exposure accelerates the emergence of antimicrobial resistance (2), antimicrobial stewardship is being increasingly emphasized. The key actions of antimicrobial stewardship in ICUs are optimizing antibiotic choice and minimizing the unnecessary administration of antibiotics.

Antimicrobial de-escalation is one part of an established strategy to optimize antibiotics. Numerous observational studies have elucidated the efficacy of antimicrobial deescalation over the past decade. Recently, several systematic reviews showed that antimicrobial de-escalation reduced the use of broad-spectrum antibiotics without compromising patients' outcomes in critically ill settings (3-5). Minimizing unnecessary antibiotic administration is another essential element of antimicrobial stewardship in ICUs. For confirmed infections, limiting the duration of antibiotic administration may be an effective strategy. Recent studies suggested that the duration of therapy for most infections in critically ill patients can be reduced to 7 days or less (6). To determine the timing of antibiotic cessation, biomarkers such as procalcitonin or C-reactive protein can be used. Several randomized studies clarified that a procalcitoninguided algorithm could reduce the duration of therapy for confirmed sepsis without worsening mortality (7). Another strategy for minimizing unnecessary antibiotic administration may be early discontinuation of antibiotics in patients in whom infection cannot be confirmed. As it is often difficult to distinguish sepsis from other noninfectious inflammatory conditions, antibiotics are frequently used for patients with presumed-but not confirmed-sepsis. A previous study reported that about $40 \%$ of patients diagnosed with sepsis who were admitted to ICUs did not actually have infections (8). To avoid unnecessary antibiotic exposure to patients without infections, it is important to confirm whether patients with presumed sepsis truly have infections. So far, however, there are few studies trying to rule out infections for presumed sepsis in the early phase of treatment.

To conquer these clinical questions mentioned above, Hellyer et al. (9) conducted a randomized controlled trial to evaluate whether the measurement of IL- $1 \beta$ and IL-8 in bronchoalveolar lavage fluid (BALF) could be used to improve antibiotic stewardship in patients with suspected ventilator-associated pneumonia (VAP). They enrolled 209 patients with suspected VAP who were assigned to a biomarker-guided recommendation group $(n=103)$ or routine use of antibiotics group $(n=106)$ in the intention-to-treat analysis. A protocolized bronchoscopy and bronchoalveolar lavage (BAL) were performed in all randomly assigned patients. In the biomarker-guided recommendation group, physicians were advised to consider discontinuation of antibiotics if the results were below the cut-off values for the biomarkers. In the routine use of antibiotics group, each patient received routine use of 
antibiotics. Although a previous study revealed that the negative predictive value of the test for microbiologically confirmed VAP was estimated at $1.0(10)$, the biomarkerguided recommendation on antibiotics did not achieve significant improvement of antibiotic-free days as a primary outcome, either in the intention-to-treat $(\mathrm{P}=0.58)$ or perprotocol analyses $(\mathrm{P}=0.28)$. The authors also found no differences for any of the secondary outcomes, including antibiotic-associated infections and 28-day mortality, between the groups.

The reported trial was unique and challenged clinical research to restrict the overuse of antibiotic agents. Evaluating IL- $1 \beta$ and IL- 8 concentrations in BALF was a novel method to exclude microbiologically confirmed VAP with high negative predictive value on the day of the examination. However, the reduction in antibiotics was not achieved due to several assumed factors. First, the BAL assays in this trial were frequently of low quality, and thus there were more unsuccessful assays in the present study than in the previous study [22/103 (21.4\%) vs. $7 / 157$ $(4.5 \%)]$. Differences in the physicians' proficiency with bronchoscopic technique could also influence the rates of unsuccessful assays. The physicians managing the patients with VAP could not be well-trained in bronchoscopic techniques. A nationwide survey of the diagnosis and management of VAP revealed that $24.5 \%$ of respondents described inadequate training in bronchoscopy, and $9.5 \%$ had no training at all (11). In fact, 7 institutions in the present study reported very infrequent or no use of BAL in the usual diagnosis of suspected VAP. Second, there were fewer patients with low IL- $1 \beta$ and IL- 8 concentrations in BALF than those in the previous study [17/103 (16.5\%) vs. $43 / 150(28.7 \%)]$. Due to the small population with values below the cut-off, the study did not have enough power to detect the superiority of the intervention. Finally, and most importantly in our view, the biomarker-guided recommendation on antibiotics was obeyed in only 4 of 17 cases $(24 \%)$, even though every physician was educated on the high negative predictive value of the test for confirming VAP. Moreover, the physicians in the present study referred not only to the protocolized suggestion based on the biomarkers (intervention), but also to the BALF culture (standard reference of VAP diagnosis). These physicians' preferences for antibiotic choice may have diluted the positive impact of interventions on the outcomes. A similar tendency was observed in another randomized control trial evaluating the efficacy of procalcitonin-guided antibiotic treatment in critically ill patients with VAP. Discouragement of the start of antibiotics and recommendations to complete antibiotics were often rejected by physicians if their patients were clinically unstable (12). The fact that recommendations to discontinue antibiotics in this trial were refused indicates difficulty in the management of suspected hospital-acquired infection in critically ill settings. Various invasions against patients make it difficult to diagnose infection correctly. Moreover, delayed or inappropriate antibiotic therapy in severely ill patients may be crucial $(13,14)$. Inherent concern about potential infection affects the physician's decision to prescribe antibiotics. Therefore, to overcome physicians' concern and change their behavior, a validation study is needed to assess whether a biomarker-guided discontinuation strategy has no detrimental effects on patient outcomes.

Optimizing antibiotic choice and minimizing the unnecessary administration of antibiotics are two of the most important fundamental actions of antimicrobial stewardship in ICUs. Further clinical trials of both actions must be continued to confront this potentially catastrophic world health problem. Currently, we are conducting a randomized controlled trial to evaluate whether Gram stain-guided antibiotic therapy restricts the overuse of broad-spectrum antibiotics without impairing patient outcomes in patients with VAP (15). Antibiotic choice based on estimation of causative pathogens is thought to be an essential strategy to optimize antibiotics. Gram staining is a traditional method, but we have demonstrated the efficacy of Gram staining on guiding appropriate antibiotic choice $(16,17)$. Clinical trials that improve antimicrobial stewardship will be the driving force to overcome the rapid pandemic spread of antimicrobial resistance throughout the world.

\section{Acknowledgments}

Funding: None.

\section{Footnote}

Provenance and Peer Review: This article was commissioned by the editorial office, Annals of Translational Medicine. The article did not undergo external peer review.

Conflicts of Interest: All authors have completed the ICMJE uniform disclosure form (available at http://dx.doi. org/10.21037/atm-20-2421). The authors have no conflicts of interest to declare. 
Ethical Statement: The authors are accountable for all aspects of the work in ensuring that questions related to the accuracy or integrity of any part of the work are appropriately investigated and resolved.

Open Access Statement: This is an Open Access article distributed in accordance with the Creative Commons Attribution-NonCommercial-NoDerivs 4.0 International License (CC BY-NC-ND 4.0), which permits the noncommercial replication and distribution of the article with the strict proviso that no changes or edits are made and the original work is properly cited (including links to both the formal publication through the relevant DOI and the license). See: https://creativecommons.org/licenses/by-nc-nd/4.0/.

\section{References}

1. Vincent JL, Rello J, Marshall J, et al. International study of the prevalence and outcomes of infection in intensive care units. JAMA 2009;302:2323-9.

2. Tacconelli E. Antimicrobial use: risk driver of multidrug resistant microorganisms in healthcare settings. Curr Opin Infect Dis 2009;22:352-8.

3. Tabah A, Cotta MO, Garnacho-Montero J, et al. A Systematic Review of the Definitions, Determinants, and Clinical Outcomes of Antimicrobial De-escalation in the Intensive Care Unit. Clin Infect Dis 2016;62:1009-17.

4. Tabah A, Bassetti M, Kollef MH, et al. Antimicrobial deescalation in critically ill patients: a position statement from a task force of the European Society of Intensive Care Medicine (ESICM) and European Society of Clinical Microbiology and Infectious Diseases (ESCMID) Critically Ill Patients Study Group (ESGCIP). Intensive Care Med 2020;46:245-65.

5. Ambaras Khan R, Aziz Z. Antibiotic de-escalation in patients with pneumonia in the intensive care unit: A systematic review and meta-analysis. Int J Clin Pract 2018;72:e13245.

6. De Waele JJ, Martin-Loeches I. Optimal duration of antibiotic treatment in Gram-negative infections. Curr Opin Infect Dis 2018;31:606-11.

7. Pepper DJ, Sun J, Rhee C, et al. Procalcitonin-Guided Antibiotic Discontinuation and Mortality in Critically Ill Adults: A Systematic Review and Meta-analysis. Chest 2019;155:1109-18.

8. Klein Klouwenberg PM, Cremer OL, van Vught LA, et al. Likelihood of infection in patients with presumed sepsis at the time of intensive care unit admission: a cohort study. Crit Care 2015;19:319.

9. Hellyer TP, McAuley DF, Walsh TS, et al. Biomarkerguided antibiotic stewardship in suspected ventilatorassociated pneumonia (VAPrapid2): a randomised controlled trial and process evaluation. Lancet Respir Med 2020;8:182-91.

10. Hellyer TP, Morris AC, McAuley DF, et al. Diagnostic accuracy of pulmonary host inflammatory mediators in the exclusion of ventilator-acquired pneumonia. Thorax 2015;70:41-7.

11. Browne E, Hellyer TP, Baudouin SV, et al. A national survey of the diagnosis and management of suspected ventilator-associated pneumonia. BMJ Open Respir Res 2014;1:e000066.

12. Bouadma L, Luyt CE, Tubach F, et al. Use of procalcitonin to reduce patients' exposure to antibiotics in intensive care units (PRORATA trial): a multicentre randomised controlled trial. Lancet 2010;375:463-74.

13. Dupont H, Mentec H, Sollet JP, et al. Impact of appropriateness of initial antibiotic therapy on the outcome of ventilator-associated pneumonia. Intensive Care Med 2001;27:355-62.

14. Kollef MH, Sherman G, Ward S, et al. Inadequate antimicrobial treatment of infections: a risk factor for hospital mortality among critically ill patients. Chest 1999;115:462-74.

15. Yoshimura J, Yamakawa K, Kinoshita T, et al. GRam stain-guided Antibiotics ChoicE for Ventilator-Associated Pneumonia (GRACE-VAP) trial: rationale and study protocol for a randomised controlled trial. Trials 2018;19:614.

16. Yoshimura J, Kinoshita T, Yamakawa K, et al. Impact of Gram stain results on initial treatment selection in patients with ventilator-associated pneumonia: a retrospective analysis of two treatment algorithms. Crit Care 2017;21:156.

17. Yoshimura J, Yamakawa K, Kinoshita T, et al. Gram stainguided antibiotic choice: a GRACEful method to safely restrict overuse of broad-spectrum antibiotic agents. Crit Care 2018;22:338.

Cite this article as: Yoshimura J, Hashimoto H, Yamakawa K. Antibiotic stewardship in critically ill patients with suspected ventilator-associated pneumonia. Ann Transl Med 2020;8(20):1329. doi: $10.21037 / \mathrm{atm}-20-2421$ 